\title{
CONSTRAINTS ON AND ALTERNATIVES TO A MASSIVE BLACK HOLE AT THE GALACTIC CENTER
}

\author{
Leonid M. Ozernoy \\ Harvard-Smithsonian Center for Astrophysics \\ Cambridge, MA 02138
}

\begin{abstract}
Considerations are presented which could serve as nourishment for a "devil's advocate" with regard to the concept of a very massive $\left(\sim 10^{6} \mathrm{M}_{\odot}\right)$ black hole at the center of the Galaxy. Constraints on the BH mass given by various processes are summarized. Most attention is paid to a novel probe of the black hole by means of a "wind diagnostic," i.e. by accounting for interaction of the BH with the wind responsible for the broad line region at the Galactic Center. All available data taken together do not require a very high mass for the $\mathrm{BH}$, but a moderately massive black hole currently seems to present the prime candidacy from several alternatives.
\end{abstract}

\section{INTRODUCTION}

The prediction of a compact radio source at the Galactic Center as a signature of the massive black hole possibly located there (Lynden-Bell and Rees 1971) which was done before the discovery of Sgr A* (Balick and Brown 1974) still remains fairly impressive evidence for the existence of a $\mathrm{BH}$ there. Curiously enough, however, both the initial arguments on which the prediction was based and the subsequent observational data do not seem at present to be as convincing as they should be.

First, a belief that almost every galaxy should harbor a massive $\mathrm{BH}$ at the galactic center has to be confronted with a counter-argument that the galaxies, depending on their initial conditions, might have different evolutionary histories. An apparent inevitability of $\mathrm{BH}$ formation which has been presented on the well-known "flow diagram" (Rees 1984) is partly undermined by the findings that some processes tend to prevent the $\mathrm{BH}$ formation while others act to retard a rapid growth of the $\mathrm{BH}$ (for a review, see Ozernoy 1988).

Second, the dynamical evidence for a massive $\left(\sim 10^{6} \mathrm{M}_{\odot}\right) \mathrm{BH}$ at the Galactic Center, that follows from the ionized gas data seems to remain still "suggestive but not compelling" (Genzel and Townes 1987). The evidence is based on simple gravitationally dominating orbit models for the gaseous filaments in the vicinity of Sgr A* (Serabyn et al. 1988). These models do not envisage non-gravitational (such as magnetic) forces on (as well as possible non-stationary motions of) the filaments. Neither is the offset of Sgr $A^{*}$ from IRS 16 (which is believed to contain the central dense star cluster) accounted for in the existing models. Radial distribution of the stellar velocity dispersion would be a good indicator of the gravitational field but these data seem to be yet incomplete and partly controversial (Rieke and Rieke 1988, McGinn el al. 1988).

Thus the fact that any unambiguous evidence for a massive BH at the Galactic Center is still "conspicuous by its absence" seems to be a serious defect of that concept. In this 
situation, many attempts to constrain the basic $\mathrm{BH}$ parameter such as the accretion rate and the BH mass have been undertaken. The present paper is aimed at discussing just those approaches. After a brief review of earlier work on the subject (Sec. 2) I shall concentrate on some novel aspects of the wind diagnostic of a black hole (Sec. 3) and its applications to the BH at the Galactic Center (Sec. 4). Models alternative to a massive BH are outlined in Sec. 5. Some observational targets which could stimulate further theoretical work are listed in Sec. 6.

\section{EARLIER WORK}

The first attempt to constrain the BH mass was done by using the observed luminosity of the Galactic Center as an upper limit to the mass-dependent accretion luminosity that results from the tidal disruption of stars passing by the BH (Hills 1975, Ozernoy 1976). Subsequent, more sophisticated models accounted for either secular (Dokuchaev and Ozernoy 1977) or short-term accretion luminosity evolution (Gurzadyan and Ozernoy 1980 ) in order to put more realistic upper limits to the BH mass. Although some quantitative details in this approach are controversial (Rees 1982) some attempts to use it in a similar way are still continuing (Lacy et al. 1982, Sanders 1984).

Since it has been found that the position of Sgr $\mathrm{A}^{*}$ on the sky does not coincide with any bright part of IRS 16 where the dense compact star cluster is thought to be located (see below), this approach does not look to be as attractive as before. Three other ways to constrain the BH mass have been proposed: (1) dynamical implications of the displacement between Sgr A* and IRS 16, (2) creation of $e^{ \pm}$pairs, and (3) the central outflow of matter. Let us discuss briefly the main results of each of these lines of attack (for more detail, see Ozernoy 1987).

\subsection{Displacement of Sgr A* from IRS 16}

This approach (Allen and Sanders 1986, Ozernoy 1982, 1987) is based on the fact that a BH of mass $M_{h}$ placed into a dense stellar system of mass $M_{*} \gg M_{h}$ should move in response to gravitational perturbations from stars mostly unbound to the BH. The mean distance of the BH from the system's center is expected to be $\langle d\rangle=\left(\pi m_{*} / 6 M_{h}\right)^{1 / 2} r_{c}$, $m_{*}$ being the characteristic stellar mass and $r_{c}$ being the core radius (Bahcall and Wolf 1976, Lin and Tremaine 1980). For the Galactic Center's BH it would give

$$
M_{h} \simeq 100 \mathrm{M}_{\odot}\left(\frac{\theta}{0 . " 5}\right)^{-2}\left(\frac{r_{c}}{0.5 \mathrm{pc}}\right)^{2}\left(\frac{m *}{0.5 \mathrm{M}_{\odot}}\right) .
$$

Equipartition in energy between the stars and the $\mathrm{BH}$ would give the latter a characteristic peculiar velocity $v_{h} \sim\left(m_{*} / M_{h}\right)^{1 / 2} v_{*} \sim 10 \mathrm{~km} / \mathrm{s}$, which is still consistent with an upper limit $\sim 40 \mathrm{~km} / \mathrm{s}$ for proper motion of Sgr A* (Backer and Sramek 1987).

This mechanism implies $\langle d\rangle \ll r_{c}$. However, kinematical evidence and especially the most recent lunar occultation data both indicate that $r_{c}$ can be comparable to or even smaller than $d$, which invalidates Eq. (1). We will return to the case when $d \gtrsim r_{c}$ in Sec. 4. 


\subsection{Production of $e^{ \pm}$pairs by a $\mathrm{BH}$}

Lingenfelter and Ramaty (1982) and Aharonian and Ozernoy (1982, unpublished) pointed out that explaining both the annihilation line and the soft gamma-ray continuum from the Galactic Center by a massive $\left(\sim 10^{6} \mathrm{M}_{\odot}\right) \mathrm{BH}$ meets with essential difficulties. The latter authors suggested an electromagnetic cascade within radiation-dominated plasma as a probable mechanism for explaining the available data. They found that in order to provide both the positron production rate $Q_{+} \sim 2.10^{43} \mathrm{~s}^{-1}$ and gamma-ray continuum $L_{\gamma}\left(E_{\gamma}<2 \mathrm{MeV}\right) \sim 2.10^{38} \mathrm{erg} / \mathrm{s}$, the source radius needs to be sufficiently small: $R \simeq 3.10^{7} \mathrm{~cm}\left(1+\tau_{\mathrm{T}}\right), \tau_{T}$ being the Thompson depth. As the cascade develops closely to the gravitational radius, $R_{\text {grav }}$, it follows from the requirement $R \geq R_{\text {grav }}$ that $M_{h} \lesssim$ $(10-30) M_{\odot}$.

Although this result is model-dependent it is remarkably low to present a severe problem for a massive BH at the Galactic Center. Further work in this direction seems highly desirable.

\subsection{Mass outflow from IRS 16/Sgr A*}

Since observations of broad HeI and HI lines in the Galactic Center had indicated a possible outflow of matter from it (Hall et al. 1982, Geballe et al. 1984), an idea to use this wind as a probe of the $\mathrm{BH}$ was pointed out (Ozernoy 1984a). In order to demonstrate its potential ability let us suppose the $\mathrm{BH}$ to be the source of the wind. If the latter is driven by radiation, it requires the $\mathrm{BH}$ luminosity to be comparable to the Eddington luminosity, i.e. $L \gtrsim L_{E d d}$. As the total luminosity of the BH at the Galactic Center does not exceed $L \simeq 3.10^{40} \mathrm{erg} / \mathrm{s}$, it gives immediately a stringent upper limit to its mass:

$$
M_{h} \lesssim 300 \mathrm{M}_{\odot} \text {. }
$$

Of course, this result is model-dependent. Besides, the BH could also accrete, and not eject, the wind. Moreover, in some conditions both accretion and ejection could coexist. All this requires a more detailed consideration which is done in the following two Sections.

\section{WIND DIAGNOSTIC OF BLACK HOLE}

\subsection{Observations}

Recent data on the Broad Line Region (BLR) in the Galactic Center and especially further prospects in this field make possible both a qualitative and quantitative diagnostic of the black hole as a possible ejector or accretor of the BLR gas. Before describing this dignostic, it seems useful to summarize the most important findings related to the BLR (Geballe et al. 1987):

(a) the BLR is extended and approximately symmetric;

(b) the high velocity gas (as seen by means of redshifted $\mathrm{Br} \alpha$ line) peaks on IRS 16, with the peak closest to IRS 16C, and is not peaked or centered at Sgr A*;

(c) a large ( 3 arc sec) spatial extent of the BLR implies that what is seen is not an immediate vicinity of a compact object responsible for infall onto or outflow from it; 
(d) the BLR is best explained as an extended outflow (wind) and not as an infall since the wind accounts most naturally for the existence of the central cavity and of shocked $\mathrm{H}_{2}$ at its edges (Gatley et al. 1984, Geballe et al. 1984);

(e) the inferred wind parameters are: characteristic radius $R \simeq 0.03 \mathrm{pc}$, gas density at this radius $n \simeq 2.10^{4} \mathrm{~cm}^{-3}$, wind velocity $v \simeq 700 \mathrm{~km} / \mathrm{s}$, and mass-loss rate $\dot{M} \simeq 4.10^{-3} \mathrm{M}_{\odot} / \mathrm{yr}$.

Some of these features (and presumably all the figures!) need to be confirmed by further observations. A serious alternative to global nuclear wind (due to either loss of mass by the core stars or outflow of matter from the $\mathrm{BH}$ ) would be local inhomogeneity such as a local superposition of individual stellar winds. While the resolving of this key issue remains a challenge for intense experimental efforts (Lacy et al. 1988) we will pay attention to the case of the global nuclear wind. The two possible origins of the wind (stars or the $\mathrm{BH}$ ), and their implications for probing the $\mathrm{BH}$, are the subject of the following analysis.

Available data, such as they are, do not allow the choice between the two possiblities suggested by Geballe et al. (1987): (i) does the observed BLR result from a primary mass loss occurring within IRS 16 or (ii) is it a secondary phenomenon resulting from a (yet undetected) ultra high-velocity wind that interacts with the ambient gas? Correspondingly, two different scenarios are possible (both assume $\mathrm{Sgr} \mathrm{A}^{*}$ to be a $\mathrm{BH}$ ):

- Scenario I. Sgr A* intercepts a part of the wind (whose origin will be discussed later on) and is an accretor;

- Scenario II. Sgr A* is a probable ejector of ultrahigh velocity wind.

\subsection{The Models}

Although both the cases of pure accretion and pure ejection are the easiest for analysis, they are hardly applicable to a real situation unless it is an extreme one. Generally, accretion onto a $\mathrm{BH}$ and ejection of matter from it are not mutually exclusive. Let us consider, as an example, axisymmetric accretion. In the course of accretion, some nonhydrodynamical mechanisms can generate an outflow of matter from the BH in the form of a jet or a wind (hereinafter we shall refer to it as BH wind in order not to confuse it with an inflowing wind).

For simplicity, we shall assume the BH wind to be spherically-symmetric. According to a general physical picture of interaction between an axisymmetric accretion flow onto a compact object emitting a spherical wind (for a review, see Kennel et al. 1983 and refs. therein) one expects a bow shock appearing at some distance from the $\mathrm{BH}$, whose value contains valuable information on the parameters of the process.

This is evident when we use a simple wind/accretion model based on the following assumptions: (i) inside the accretion radius, $r<r_{a}$, the accretion flow is radial so that its velocity and density are changed as at the free-fall; (ii) beyond the accretion radius, the density and the pressure of the accreting gas are constant ( $\rho_{\infty}$ and $p_{\infty}$, correspondingly); (iii) the $\mathrm{BH}$ wind could be emitted from some radius $r_{0}$ with a velocity exceeding the 
local escape velocity so that the dynamical pressure of the BH wind changes at $r>r_{0}$ as $p_{w}=p_{0}\left(r / r_{0}\right)^{-2}$.

Under these assumptions, a straightforward procedure leads to the following equation for the stand-off distance $r_{s w}$ at which $p_{a}=p_{w}$ (cf. Shima et al. 1986):

$$
\begin{gathered}
A+1+A\left(\frac{r_{a}}{r_{s w}}\right)=B\left(\frac{r_{a}}{r_{s w}}\right)^{2} \text { if } r_{s w}>r_{a} \\
A+5+(A-4)\left(\frac{r_{a}}{r_{s w}}\right)=B\left(\frac{r_{a}}{r_{s w}}\right)^{1 / 2} \text { if } r_{s w}<r_{a} .
\end{gathered}
$$

Here $A=\gamma \mathcal{M}^{2}$ (M $=v / c_{\infty}$ being the Mach number of the upstream gas) and $B=$ $\delta^{-2}\left(\rho_{0} c_{0}^{2} / \rho_{\infty} c_{\infty}^{2}\right)$ with $\delta=r_{a} / r_{0}$. A combination of accreting flow with emitting wind is possible only if $B>B_{\text {crit }}, B_{\text {crit }} \approx 1.6 A$ for $A \gg 5$ (Shima et al. 1986).

When neglecting numerical coefficients of the order of unity this condition reads $r_{0} / r_{a} \gtrsim \mathcal{M}\left(p_{\infty} / p_{0}\right)^{1 / 2}$. Therefore, an emitting wind could be formed only at sufficiently large $p_{0}$. Depending on $p_{0}$ and $r_{0}$, two different regimes, one with $r_{s w}>r_{a}$ and another with $r_{s w}<r_{a}$, i.e. with or without a BH wind beyond $r_{a}$, are possible. Consider them in turn.

- $r_{s w}>r_{a}$. Eq. (3a) has the root

$$
r_{s w}=r_{a} \sqrt{B /(A+1)} \approx r_{0} \mathcal{M}^{-1}\left(p_{0} / p_{\infty}\right)^{1 / 2},
$$

which is independent of $r_{a}$ and therefore of $M_{h}$. The larger $p_{0}$, the larger is the stand-off distance at a given $p_{\infty}$ and $v_{\infty}$.

- $r_{s w}<r_{a}$. Eq. (3b) has the root

$$
r_{s w}=r_{a}\left(\frac{A-4}{B}\right)^{2} \approx r_{a}\left(\frac{r_{a}}{r_{0}}\right)^{4} \gamma^{2} \mathcal{M}^{2}\left(\frac{p_{\infty}}{p_{0}}\right)^{2} .
$$

In the case of a stellar wind interacting with an accretion flow, this root corresponds to an unstable solution since any slight departure from $r_{s w}$ enhances it because of $\left|\nabla p_{w}\right|>$ $\left|\nabla p_{a}\right|$. In the case of a BH wind the situation could be different since both $r_{0}$ and $p_{0}$ are not fixed here (in contrast to the case of stellar wind) and are dependent, in an as yet unknown way, on parameters of accretion. This may result in an oscillating solution. Further work should clarify this point.

If Eq. (5) gives a stable (or quasi-stable) root, it could be used to determine the $\mathrm{BH}$ mass whenever $r_{0}$ and $p_{0}$ are known from some independent data. Since $r_{0} \leq r_{s w} \leq r_{a}$, a simple estimate of $\mathrm{BH}$ mass is given by (numerical factors of order of unity are disregarded here):

$$
\frac{r_{0} v_{\infty}^{2}}{G}\left(\frac{p_{0}}{p_{\infty}}\right)^{2 / 5} \mathcal{M}^{-2 / 5}<M_{h}<\frac{r_{0} v_{\infty}^{2}}{G}\left(\frac{p_{0}}{p_{\infty}}\right)^{1 / 2} \mathcal{M}^{-1 / 2} .
$$




\section{APPLICATION TO SGR A*}

\subsection{Scenario I: Sgr $\mathrm{A}^{*}$ as an Accretor}

Let the wind passing by $\mathrm{Sgr} \mathrm{A}^{*}$ (the latter is assumed to be a BH of mass $M_{h}$ ) be captured with the rate $\dot{M} \propto M_{h}^{2} n_{\infty} / v_{\infty}^{3}$ where $n_{\infty}$ and $v_{\infty}$ are, correspondingly, the wind density and velocity observed far from the BH. In order that the persistent accretion luminosity should not exceed, at any wavelength, the observed luminosity, $L_{o b s}$, the value of $\dot{M}$ should be less than a certain limit, which immediately gives an upper limit to $M_{h}$.

To specify, we use a standard rate of accretion onto a moving BH in a uniform medium (Shapiro and Teukolsky 1983) which for supersonic flow is reduced to $\dot{M}=$ $4 \pi \tilde{\lambda} G^{2} M_{h}^{2} \rho_{\infty} / v_{\infty}^{3}, \tilde{\lambda} \sim 1$ being a constant. The proper velocity of Sgr A*, whose upper limit is much smaller than $v_{\infty}$, can be neglected in this expression. Since $\dot{M}$ should not exceed $L_{o b s} / \epsilon c^{2}$, one gets

$$
M_{h}<1.1 \cdot 10^{5} \mathrm{M}_{\odot} \frac{\left(L_{o b s} / 10^{7} \mathrm{~L}_{\odot}\right)^{1 / 2}\left(v_{\infty} / 700 \mathrm{~km} \mathrm{~s}^{-1}\right)^{3 / 2}}{(\epsilon / 0.1)^{1 / 2}\left(n_{\infty} / 2.10^{4} \mathrm{~cm}^{-3}\right)^{1 / 2}}
$$

A few comments on this result seem to be relevant. (a) It has been assumed here that the $\mathrm{BH}$ contribution to the observed IR luminosity of the Galactic Center does not exceed $3 \cdot 10^{40} \mathrm{erg} / \mathrm{s}$. But in reality IRS 16 (whose luminosity was used) and Sgr A* are spatially separated so that the contribution of $\mathrm{Sgr} \mathrm{A}^{*}$ to $L_{o b s}$ and therefore the corresponding upper limit to $M_{h}$ should be smaller. (b) The integral (bolometric) luminosity enters Eq. (7). Meanwhile the spectral luminosity like that used by Lacy $e$ t al. (1982), being compared with an expected disk luminosity would give, in principle, an even more stringent upper limit to $M_{h}$. But this approach should wait for further BH models of Sgr A*, both more detailed and free of the current defaults. (c) The upper limit to $M_{h}$ given by Eq. (7) has been derived for a disk accretion whose efficiency $\epsilon \sim 0.1$. Although the Bondi accretion is quasi-radial at $r<r_{a}$, accounting for magnetic fields, turbulence, etc. could hardly result in $\epsilon \ll 0.1$. Happily, a weak dependence of Eq. (7) on $\epsilon$ makes any uncertainties in $\epsilon$ not very important.

Another way of estimating the BH mass follows from an additional assumption about the value of stand-off distance $r_{s w}$, where the pressure of inflowing wind is balanced by that of the emitting BH wind. One extreme for this assumption would be $r_{s w}=r_{0}$, and the other would be $r_{s w}=r_{a}$. The values $r_{0}$ and $p_{0}$ can be taken from synchrotron models of Sgr $A^{*}$. Such a model, which is consistent with available observational constraints on Sgr $\mathrm{A}^{*}$, gives the radius of the source $R \simeq 10^{14} \mathrm{~cm}$ and the pressure of relativistic electrons there $p \simeq 1$ dyne $/ \mathrm{cm}^{2}$. Then Eq. (6) gives $M_{h} \simeq 4 \cdot 10^{5} \mathrm{M}_{\odot}$ if $r_{s w} \simeq r_{0}=$ $R$ and $M_{h} \simeq 1.8 \cdot 10^{3} \mathrm{M}_{\odot}$ if $r_{s w} \simeq r_{a}=R$. Both these estimates (which are consistent with the upper limit given by Eq. (7)) appear to be smaller than the currently fashionable value of $M_{h} \simeq$ few $10^{6} \mathrm{M}_{\odot}$. Further observations hopefully would make this way of constraining the Sgr $\mathrm{A}^{*}$ mass more reliable.

\subsection{Scenario II: Sgr $A^{*}$ as an Ejector}

Let $\mathrm{Sgr} \mathrm{A}^{*}$ be the primary source of a yet unseen, high-velocity wind responsible for the 
observed BLR features. Absence of any direct data makes this scenario highly speculative so that it would be premature to go into much detail. Here I will consider briefly two more or less natural versions of this scenario.

\section{- Radiative-Driven Outflow from the BH}

In order to induce a radiative-driven wind from a $\mathrm{BH}$, its total luminosity should be at least comparable with the Eddington luminosity (see Sec. 2 for detail) which gives $M_{h} \lesssim 300 \mathrm{M}_{\odot}$ for Sgr A*. [This upper limit would be even improved when further high-resolution measurements extract Sgr $\mathrm{A}^{*}$ contribution into the total IR flux from IRS $16 / \mathrm{Sgr} A^{*}$ complex.]

\section{- Ejection of the Debris of a Tidally Disrupted Star}

The tidal break-up of a star in the vicinity of a massive $\left(\sim 10^{6} \mathrm{M}_{\odot}\right)$ BH may result in ejection of gas with a very high velocity, up to $5000 \mathrm{~km} / \mathrm{s}$ (Lacy et al. 1982; Hills 1988; Rees 1988) which can serve as a source for the secondary wind at the Galactic Center. However, a displacement between Sgr A* and any of discrete sources comprising IRS 16 complex indicates that $\mathrm{Sgr} \mathrm{A}^{*}$ is apparently located far outside the star density peak. In this case, the tidal disruption of stars by the BH should be a much more rare event, which makes this picture less attractive than it could be.

\subsection{Some Comments}

Before any general conclusions from the wind diagnostic of the BH at the Galactic Center can be made, it is appropriate to remember at this point that even Scenario I, which is both less speculative and more elaborated, is based on a fundamental assumption: When considering the Bondi accretion onto the $\mathrm{BH}$, the influence of individual stellar winds onto the accretion was ignored. Moreover, so was the possibility that these winds, and not a global one, might locally govern the accretion. If a local wind were dominating in the vicinity of the $\mathrm{BH}$, a constraint to its mass would be weaker than the upper limit (7).

On the other hand, if there were no global wind at all, a new factor would appear which could make that constraint even more stringent. In this case, some rarefied, pervasive, steady gas could exist in the vicinity of Sgr $\mathrm{A}^{*}$. Indeed, available data based on the observed SIII and [OIII] line ratios in an extended region do not exclude the presence of a pervasive, steady gas there with temperature $T \simeq 7500 \mathrm{~K}$ and density $n_{g} \sim 2 \cdot 10^{3} \mathrm{~cm}^{-3}$. Such gas could be accreted onto the BH within an accretion radius that is comparable to the size of the central "cavity" within the molecular torus. At a given limiting accretion luminosity, the upper limit to the BH mass would be smaller than that given by Eq. (7) by a factor of $\left(v_{\infty} / a_{\infty}\right)^{3 / 2}\left(n_{g} / n\right)_{\infty}^{1 / 2} \sim 300, a$ being the sound velocity. The absence of a global nuclear wind at the Galactic Center could make life for proponents of the massive $\mathrm{BH}$ more troublesome!

Although both scenarios demonstrate how a wind in the Galactic Center can be used as a probe of the $\mathrm{BH}$, they leave unsolved such an important problem as why Sgr $\mathrm{A}^{*}$ is located off IRS 16 where both the kinematical center of the ionized gas streamers and presumably the dynamical center of the Galaxy are situated. This issue seems even more 
challenging since recent lunar accultation data (Simons et al. 1988) have indicated all the components of IRS 16 be point-like; no extended source that could be identified as the dynamical core of the Galaxy has been found ( $c f$. Adams et al. 1988). Such a puzzling situation would be explained by a displaced position of Sgr $A^{*}$ if the tidal radius of the core were less than the core radius. To do this, the mass of Sgr $A^{*}$ as a BH does not need to be tremendously large (see below). If so, is the apparent absence of the dynamical core indirect evidence that Sgr A* be such a BH?

In this case, the displacement between Sgr A* and IRS 16 appears to be much larger than the core radius. Meanwhile, any gravitational interactions of a black hole with surrounding stars would leave the BH within the core (see Sec. 2).

A large displacement between the $\mathrm{BH}$ and the central core seems to be possible in one of the following cases:

(a) the BH has been ejected from the core due to the "rocket effect" (Saslaw and Whittle 1988);

(b) the $\mathrm{BH}$ has been formed outside the core, i.e., not in the place of the highest star density;

(c) Sgr $\mathrm{A}^{*}$ is not a $\mathrm{BH}$.

For case (a), a conservative upper limit to the $\mathrm{BH}$ mass is given by $M_{h} \lesssim M_{c}, M_{c}$ being the core mass. Since the lunar occultation data give a very stringent upper limit to the core size for any of IRS 16 component ( $\$ 0 . " 05), M_{c}$ appears to be quite small (cf. Bailey 1980) and may not characterize its initial value. On the other hand, the disruptive action of the $\mathrm{BH}$ onto the core enables us to get a lower limit to $M_{h}$ given by

$$
M_{h}>20(\theta / 0 . " 5)^{3}\left(m_{*} / 0.5 \mathrm{M}_{\odot}\right)\left(n * / 10^{6} \mathrm{pc}^{-3}\right) \mathrm{M}_{\odot}
$$

$\theta \sim 0.5$ arc sec being the distance between Sgr A* and a (yet unknown) position of the dynamical core, and $n *$ being the star density in the core. When determined by further observations, the parameters of the core could give valuable constraints to the $\mathrm{BH}$ mass.

Case (b) seems possible only if $M_{h} \lesssim 30-300 \mathrm{M}_{\odot}$, i.e., if the $\mathrm{BH}$ has been formed as a result of the collapse of a massive (or very massive) star.

Case (c), considered in detail in the next section, and case (b) both leave open the question of whether either case is compatible with a small $r_{c}$.

A balanced conclusion consistent both with the wind diagnostic and the displacement of Sgr A* would be the following: they do not require the presence of a huge $\left(\sim 10^{6} \mathrm{M}_{\odot}\right)$ $\mathrm{BH}$ at the Galactic Center.

But if not such a monster, what else?

\section{ALTERNATIVE MODELS}

\subsection{Young Pulsar (YP)}

Since the Galactic Center is considered as a region of intensive star formation, a young 
pulsar as an evolutionary remnant of a massive star has been considered for years to explain the nature of Sgr A* (Davies et al. 1976; Ozernoy and Shishov 1977; Reynolds and McKee 1980; Mastichiadis et al. 1987; Engelke 1988). Main hopes have been connected with the potential capability of a young pulsar to produce $e^{ \pm}$pairs. However, detailed models of $e^{ \pm}$pair creation in the pulsar magnetosphere demonstrate (e.g. Ruderman and Sutherland 1975) that positron output is even smaller than in the Sturrock (1971) model, i.e. less than $10^{41} \mathrm{~s}^{-1}$. The reason is that $e^{ \pm}$pair creation leads to a partial screening of the polar gap and to a decreasing of it to the free path length, $l_{\gamma}$, for the curvature radiation quanta which results in a potential drop of the pulsar gap.

Two kinds of attempts to resolve that problem have been undertaken:

(a) due to resonance effects near the threshold of $e^{ \pm}$pair creation in a sufficiently strong magnetic field (Shabad and Usov 1984), the value of $l_{\gamma}$ becomes comparable to the pulsar radius so that any significant screening of the gap does not occur. As a result, a young and fast rotating $\left(\Omega \sim 300 \mathrm{~s}^{-1}\right)$ as well as a sufficiently magnetized $\left(B \gtrsim 4 \cdot 10^{12} \mathrm{G}\right.$ ) pulsar would be capable of providing the necessary rate of $e^{ \pm}$pair production, although some unsettled issues still remain (Ozernoy and Usov 1982, unpublished);

(b) if a young ( $t \lesssim 200 \mathrm{yr}$ ) pulsar has a high surface temperature $\left(T \sim 10^{7} \mathrm{~K}\right)$, the interactions between its soft $\mathrm{X}$-ray photons and highly relativistic electrons could produce, via electromagnetic cascade, the $e^{ \pm}$pairs with $\gamma \sim 10^{2}-10^{3}$ (Mastichiadis et al. 1987).

A common difficulty of all YP models is that the typical pulsar velocity $v \sim 100-300$ $\mathrm{km} / \mathrm{s}$ appears incompatible with the above-mentioned very stringent upper limit to $\mathrm{Sgr}$

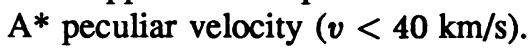

\subsection{Moderate-Mass Black Hole (MMBH)}

A suspicion that the hypothesis of a starved $\mathrm{BH}$ at the Galactic Center would meet with difficulties unless its mass is small enough (Ozernoy 1976) obtained additional support during subsequent years (Lingenfelter and Ramaty 1982; Ozernoy 1982, 1984a,b; Allen and Sanders 1986) and has been strengthened by new arguments discussed in Ozernoy (1987) and above. The advantages of a MMBH, besides being consistent with the available observational constraints, is its capability to explain the $e^{ \pm}$pair production in the Galactic Center. But it is still unclear whether MMBH could explain the radio and infrared properties of Sgr A*/IRS 16 complex.

\subsection{A Single/Multiple Very Massive Star(s) (VMS)}

The presence of a relatively young stellar population at the Galactic Center (Lebofsky $e t$ al. 1982) makes it possible that a central object could resemble $\eta$ Car or R 136a (Ozernoy $1982,1984 a, b ;$ Werner 1988), i.e. it could be a very massive single/multiple star(s). If single, such a star of the mass, say, $M=300 \mathrm{M}_{\odot}$ has luminosity $L \simeq 10^{7} \mathrm{~L}_{\odot}$, radius $R \simeq 100 R_{\odot}$, mass loss rate $\dot{M} \simeq 10^{-3} \mathrm{M}_{\odot} / \mathrm{yr}$, and lifetime $\sim 10^{6} \mathrm{yr}$ ( or more if it is being fed by a gaseous disk: see Kundt 1979, Krügel and Tutukov 1986). This makes 
possible the explanation of such features of the Galactic Center as its infrared luminosity, production of the central wind and, if VMS is magnetized (a small magnetoid/spinar), it might be a source of magnetic loops production.

\subsection{Low-Mass X-Ray Binaries (LMXB)}

Models to explain $e^{ \pm}$pair production in the Galactic Center range by now from a supermassive BH to low-mass X-ray binaries (Kluźniak et al. 1988). This is eloquent evidence that the current observational status of the issue is far from being satisfactory in order to choose between such extremes.

\section{CONCLUSIONS}

The above models have a common flaw: they are capable of explaining only some, and not all, of the properties of Sgr A*/IRS 16 complex. Even if we restrict ourselves only by its continuum radiation, the partial luminosities at different spectral ranges are explainable by different models, not by a single one:

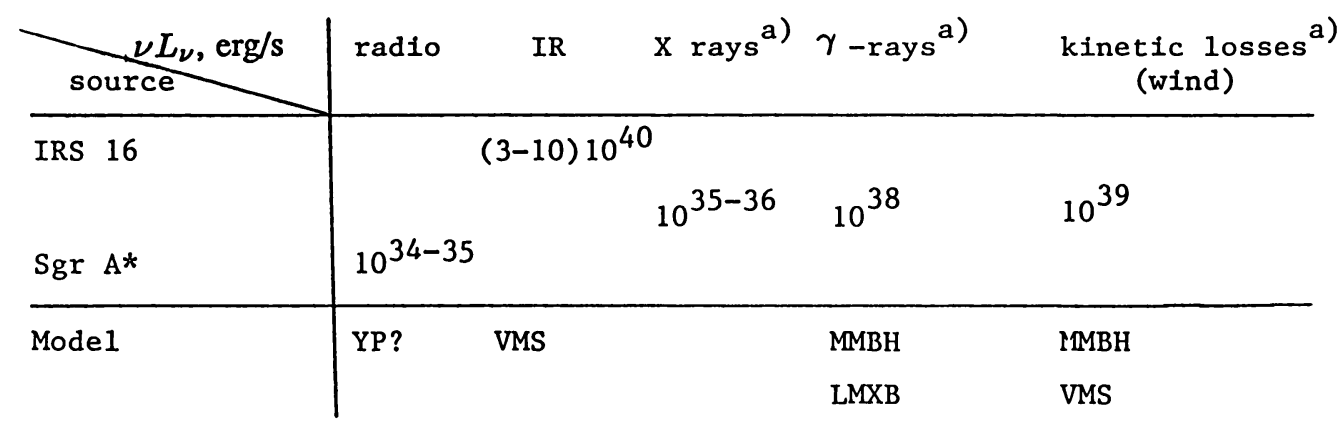

a) Location of the source unknown

A conjecture that could resolve this difficulty is that a symbiotic model, or a hybrid (such as MMBH + VMS) would explain the most essential properties of Sgr $A^{*} / \mathrm{IRS}$ 16. A possible scenario for the origin of such a hybrid as a result of a recent star burst episode has been given in Ozernoy (1984b). A black hole of a moderate mass seems to have the most credit as (a part of or even a whole) the "engine" at the Galactic Center, but reliable proofs of that should wait for further observational data.

In the light of what has been discussed above, a list of important observational issues whose resolution would greatly stimulate the further progress should include the following:

- Location of the dynamical center and value of the Population II core radius;

- Nature of the components of IRS 16 (multiple $v$ individual stars, peculiar velocities, spectral types, stellar winds, etc);

- A higher spatial and kinematical resolution of the broad line region in the Galactic 
Center (structure of the wind, temporal changes, and origin of the wind seem to be the key items);

- More about Sgr A* (variability data, millimeter VLBI observations, and mm/submm/IR spectral data all would make possible the testing of the synchrotron model of Sgr $\mathrm{A}^{*}$ as well as deriving its global parameters);

- Location, extent, and variability of the X-ray and $\gamma$-ray source(s) in the Galactic Center (this would put essential constraints on the origin of the high-energy phenomena there).

\section{ACKNOWLEDGEMENTS}

I am thankful to M. Morris and the members of the Organizing Committee who made possible my participation in such a landmark event as the present Symposium. It is a pleasure to acknowledge the valuable discussions with D. Allen, D. Backer, E. Becklin, R. Blandford, T. Geballe, R. Genzel, J. Lacy, K. Lo, M. Morris, R. Pudritz, G. Rieke, S. Phinney, C. Townes, and many other participants. I thank Ms. N.L. Snyder for help with preparing the manuscript.

\section{REFERENCES}

Adams, D. J., Becklin, E.E., Jameson, R.F., Longmore, A.J., Sandquist, Aa., and Valentijn, E.: 1988, Ap.J. 327, L65

Allen, D.A., and Sanders, R.H.: 1986, Nature 319, 191

Backer, D.C. (ed.): 1987, The Galactic Center, AIP Conf. Proc. No. 155

Backer, D.C., and Sramek, R.A.: 1987, in Backer (ed.), p.163

Bahcall, J.N., and Wolf, R.A.: 1976, Ap.J. 209, 214

Bailey, M.E.: 1980, Mon. Not. R.A.S. 190, 217

Balick, B., and Brown, R.L.: 1974, Ap.J. 194, 265

Davies, R.D., Walsh, D., and Booth, R.S.: 1976, Mon. Not. R.A.S. 177, 319

Engelke, C.W.: 1988, Ap.J. 325, 153

Dokuchaev, V.I., and Ozernoy, L.M.: 1977, Sov. Astr. Lett. 3(5),209

Gatley, I., Jones, T.J., Hyland, A.R., Beattie, D.H., and Lee, T.J.: 1984, Mon. Not. R.A.S. 210, 565

Geballe, T.R., Krisciunas, K.L., Lee, T.J., Gatley, I., Wade, R., Duncan, W.D., Garden, R., and Becklin, E.E.: 1984, Ap.J. 284, 118

Geballe, T.R., Wade, R., Krisciunas, K.L., Gatley, I., and Bird, M.C.: 1987, Ap.J. 320, 562

Genzel, R., and Townes, C.H.: 1987, Ann. Rev. Astron. Astrophys. 25, 377

Gurzadyan, V.G., and Ozernoy, L.M.: 1980, Astron. Astrophys. 86, 315

Hall, D.N.B., Kleinmann, S.G., and Scoville, N.Z.: 1982, Ap.J. 262, L53

Hills, J.: 1975, Nature 254, 295

Hills, J.: 1988, Nature 331, 687

Kennel, C.F., Fujimura, F.S., and Okamoto, I.: 1983, Geophys. Astrophys. Fluid Dynamics 26, 147

Kluźniak, W.: this Symposium

Krügel, E., and Tutukov, A.: 1986, Astron. Astrophys. 158, 367 
Kundt, W.: 1979, Ap. Sp. Sci. 62, 335

Lacy, J.H., Townes, C.H., and Hollenbach, D.J.: 1982, Ap.J. 262, 120

Lacy, J.H., Achtermann, J.M., and Bruce, D.E.: this Symposium

Lebofsky, M.J., Rieke, G.H., and Tokunaga, A.T.: 1982, ApJ. 263, 736

Lin, D.N.C., and Tremaine, S.: 1980, Ap.J. 242, 789

Lingenfelter, R.E., and Ramaty, R.: 1982, in Riegler and Blandford (eds.), p. 148

Lacy, J.H., Townes, C.H., and Hollenbach, D.J.: 1982, Ap.J. 262, 120

Lynden-Bell, D., and Rees, M.J.: 1971, Mon. Not. R.A.S. 152, 461

Mastichiadis, A., Brecher, K., and Marscher, A.P.: 1987, Ap.J. 314, 88

McGinn, M.T., Sellgren, K., Becklin, E.E., Hall, D.N.B., and Gatley, I.: this Symposium

Ozernoy, L.M.: 1976, Observatory 96, 67

Ozernoy, L.M.: 1982, "On the Nature of the Compact Source at the Galactic Center", paper presented at the XVIII General Assembly of the IAU, read by R.Ramaty

Ozernoy, L.M.: 1984a, Astron. Tsirk. No. 1342, 1

Ozernoy, L.M.: 1984b, Astron. Tsirk. No. 1349, 1

Ozernoy, L.M.: 1987, in Backer (ed.), p. 181

Ozernoy, L.M.: 1988, in Supermassive Black Holes (Ed. M. Kafatos). Cambridge Univ. Press, p. 211

Ozernoy, L.M., and Shishov, V.I.: 1977, Sov. Astr. Lett. 3( 5), 233

Rees, M.J.: 1982, in Riegler and Blandford (eds.), p. 166

Rees, M.J.: 1984, Ann. Rev. Astron. Astrophys. 22, 471

Rees, M.J.: 1988, Nature 333, 523

Reynolds, S.P., and McKee, C.F.: 1980, Ap.J. 239, 893

Riegler, G.R., and Blandford, R.D. (eds.): 1982, The Galactic Center, AIP Conf. Proc. No. 83

Rieke, G.H., and Rieke, M.J.: 1988, Preprint

Ruderman, M., and Sutherland, P.: 1975, Ap.J. 196, 51

Sanders, R.H.: 1984, Astron. Astrophys. 140, 52

Saslaw, W.C., and Whittle, M.: 1988, ApJ. 325, 142

Serabyn, E., Lacy, J.H., Townes, C.H., and Bharat, R.: 1988, Ap.J. 326, 171

Shabad, A.E., and Usov, V.V. 1984, Astrophys. Sp. Sci. 102, 327

Shapiro, S.L., and Teukolsky, S.A.: 1983, Black Holes, White Dwarfs, and Neutron Stars, Wiley-Interscience Publ.

Shima, E., Matsuda, T., and Inaguchi, T.: 1986, Mon. Not. R.A.S. 221, 687

Simons, D.A. Becklin, E.E., and Hodapp, K.-W.: this Symposium

Sturrock, P.A.: 1971, Ap.J. 164, 529

Werner, M.: this Symposium 